\title{
Stable fractal sums of pulses: the cylindrical case
}

\author{
RENATA CIOCZEK-GEORGES, 'BENOIT B. MANDELBROT, ' \\ GENNADY SAMORODNITSKY ${ }^{2}$ and MURADS. TAQQU ${ }^{3}$ \\ 'Yale University, 10 Hillhouse Avenue, New Haven CT 06250-8283, USA \\ ${ }^{2}$ Cornell Universiry, Ithaca NY 14853, USA \\ ${ }^{3}$ Boston University, 111 Cummington Street, Boston MA 0221S-2411, USA
}

A class of $\alpha$-stable, $0<\alpha<2$, processes is obtained as a sum of 'up-and-down' pulses determined by an appropriate Poisson random measure. Processes are $H$-self-affine (also frequently called 'self-similar') with $H<1 / \alpha$ and have stationary increments. Their two-dimensional dependence structure resembles that of the fractional Brownian motion (for $H<1 / 2$ ), but their sample paths are highly irregular (nowhere bounded with probability 1). Generalizations using different shapes of pulses are also discussed.

Keywords: measures of dependence; path behaviour; Poisson random measure; self-affinity; self-similarity: stable processes; stationarity of increments

\section{Introduction}

This paper presents a physically motivated construction that yields a class of self-affine stable processes (not necessarily symmetric) with stationary increments. A process $\{X(t), t \geq 0\}$ is obtained as a sum of an infinite number of pulses whose height varies by jumps, that is, discontinuities, to be called rises or falls. Consider the initial jump of a given pulse. Its time of occurrence and its height are governed by the Poisson random measure that is classically used to obtain Lévy stable motions (see, for exampie, Itô 1969). But in our construction a pulse does not ređuce to this initial jump. In the case of the simplest 'up-and-down' pulses every 'rise' (or 'fall') of a pulse is to be followed by another 'fall' ('rise') of the identical absolute size, a 'cancelling echo', which occurs after a random duration of time. General pulses may involve more than two jumps, but the ups and downs must always cancel out. This more complicated random scenario causes increments of the process $\{X(t), t \geq 0\}$ to be dependent, while the increments of the Lévy stable motion are independent.

However, the increments remain stationary, that is, $\{X(t+b)-X(b), t \geq 0\} \stackrel{\mathrm{d}}{=}$ $\{X(t)-X(0), t \geq 0\}$ for all $b>0$, where ' $=$ ' denotes equality of finite-dimensional distributions. In addition, the process is self-affine (it is also frequently called 'self-similar'), that is, there exists $H>0$, such that $\{X(a t), t \geq 0\} \stackrel{d}{=}\left\{a^{H} X(t), t \geq 0\right\}$ for all $a>0$. It is known (see Kono and Maejima 1991; or Samorodnitsky and Taqqu 1994) that for $\alpha$-stable processes, with the characteristic exponent $0<\alpha \leq 2$, the self-affinity constant $H$ must satisfy $0<H \leq \max \{1,1 / \alpha\}$. In our case $H<1 / \alpha$ and its specific value will depend on the distribution of the puise width. Levy stable motions have a single scaling exponent $H=1 / \alpha$ and the reason for introducing our $\{X(t), t \geq 0\}$ was the desire to provide a physical construction of processes for which $H \neq 1 / \alpha$. 
The one-dimensional distributions of $\{X(t), t \geq 0\}$ are symmetric $\alpha$-stable ( $\alpha S)$ and the joint multidimensional distributions are stable, but need not be symmetric. In fact, the symmetric $\{X(t), t \geq 0\}$ falls in the category of Chentsov-type processes obtained by Takenaka (1991) by means of integral geometry and extensions thereof considered by Mori and Sato (1994). While Takenaka's representation is valid only in the symmetric case, ours works both in the symmetric and non-symmetric cases. The dependence structure of Chentsov-type processes, as shown by Sato $(1991 ; 1992)$, is determined by their two-dimensional distributions. So is the dependence structure of our process $\{X(t), t \geq 0\}$, even in the non-symmetric case.

We also examine the behaviour of the normalized codifference and the normalized covariation of non-overiapping increments of $\{X(t), t \geq 0\}$. Both the codifference and the covariation extend the notion of covariance to the non-Gaussian case. For our process $\{X(t), t \geq 0\}$, the normalized codifference and covariation, as well as another measure of dependence introduced here, are all equal to the correlation of the increments of fractional Brownian motion - a self-affine Gaussian. process. If the classical terminology of the second-order processes were to be used we would say that the one-step increments of the constructed process exhibit negative global dependence. (For the analogous construction of fractional Brownian motion see Cioczek-Georges and Mandeibrot (1994a; 1994b).)

We also point out that the path behaviour of the pulse process is very irregular, contrary to previous beliefs about the process introduced by Takenaka (1991). Sample paths of $\{X(t), t \geq 0\}$ are nowhere bounded with probability 1 .

Finally we consider possible generalizations. One is in the same spirit as Takenaka's generalization of his Chentsov-type processes, that is, the time parameter becomes multidimensional and rectangular pulses change to multidimensional cylinders. In another generalization time is kept one-dimensional, but the pulse shape becomes more complicated: it consists of several falls or rises separated by random durations of time. For such processes self-affinity and stationarity of increments still hold; however, the dependence structure is much more complex.

The idea of adding up pulses to construct self-affine stable processes has aiready been investigated by Lovejoy and Mandelbrot (1985) (see also Mandelbrot 1995a; 1995b). They used two-dimensional versions of a pulse process to model rain areas and rain rate. In their construction, however, the pulses' widths are functions of the pulses' heights, yielding a process which is in the domain of attraction of Lévy stable motion. Processes with independent pulse widths and heights were considered for the first time in Mandelbrot (1984) and this memorandum inspired the present paper and its generalization in Cioczek-Georges and Mandelbrot (1995). For a general introduction to stable processes see the recent monograph of Samorodnitsky and Taqqu (1994).

We now turn to the case of up and down pulses. The pulse address space is defined as $E=\mathbb{R}_{0} \times \mathbb{R} \times \mathbb{R}_{+}$, where $\mathbb{R}_{0}=\mathbb{R} \backslash\{0\}$ and $\mathbb{R}_{+}=(0, \infty)$. Let $\mathscr{E} \equiv \mathscr{B}$ be the Borel $\sigma$-field on $E$. Consider a Poisson random measure $N$ on $(E, \mathscr{E})$ with mean $n$ given by

$$
n(\mathrm{~d} \lambda, \mathrm{d} \tau, \mathrm{d} w)= \begin{cases}c^{\prime} \lambda^{-\alpha-1} w^{-\theta-1} \mathrm{~d} \lambda \mathrm{d} \tau d w & \text { if } \lambda>0, \\ c^{\prime \prime}|\lambda|^{-\alpha-1} w^{-\theta-1} \mathrm{~d} \lambda \mathrm{d} \tau \mathrm{d} w & \text { if } \lambda<0\end{cases}
$$

for $\tau \in \mathbb{R}, w \in \mathbb{R}_{+}$, and some $0<\alpha<2,0<\theta<1, c^{\prime}, c^{\prime \prime} \geq 0, c^{\prime}+c^{\prime \prime}>0$.

Each pulse is represented in $E$ by a point with coordinates $\lambda, \tau$ and $u$, corresponding respectively 
to the vertical amplitude (height), time of birth and width (duration) of a pulse. Hence, the random measure $N$ determines the number of pulses of given heights, widths and starting times.

The process $X(t)$ at times $t>0$ is defined as the sum of the heights of ail the puises ailive at time $t$ minus the sum of the heights of all the pulses alive at time 0 . Thus, to get the value of $X(t)$, we have to add the heights of all pulses that started between 0 and $t$ and died after time $t$, and subtract the sum of the heights of the pulses that started before 0 and died between 0 and $t$. Of course, both sums could be negative because we assume that both positive and negative pulses $(\lambda>0$ or $\lambda<0)$ can occur. Note that the heights of pulses which started before 0 and died after $t$ cancel out and thus need not be included in our summation. It is also clear that we can ignore the pulses that start and end between 0 and $t$.

In fact, $X(t), t>0$ is an integral with respect to number of pulses. Its formal definition is as follows:

$$
X(t)= \begin{cases}\int_{\mathbb{R}_{u}} \int_{0}^{t} \int_{0}^{\infty} \lambda I[w>t-\tau] N(\mathrm{~d} \lambda, \mathrm{d} \tau, \mathrm{d} w) & \text { if } 0<\alpha<1, \\ -\int_{\mathbb{R}_{u}} \int_{-\infty}^{0} \int_{0}^{\infty} \lambda I\left[-\tau<w^{\prime}<t-\tau\right] N\left(\mathrm{~d} \lambda, \mathrm{d} \tau, \mathrm{d} w^{\prime}\right) & \\ \lim _{\epsilon \rightarrow 0}\left[\int_{\left(-\epsilon, \epsilon^{c}\right.} \int_{0}^{t} \int_{0}^{\infty} \lambda I[w>t-\tau] N(\mathrm{~d} \lambda, \mathrm{d} \tau, \mathrm{d} w)\right] & \\ -\int_{(-\epsilon, \epsilon)^{\mathrm{c}}} \int_{-\infty}^{0} \int_{0}^{\infty} \lambda I[-\tau<w<t-\tau] N(\mathrm{~d} \lambda, \mathrm{d} \tau, \mathrm{d} w) & \text { if } 1 \leq \alpha<2 .\end{cases}
$$

For consistency, we set $X(0) \equiv 0$.

We must show that the above integrals converge. It turns out that, in the case $1 \leq \alpha<2$. the integrals over $\mathbb{R}_{0}$ converge only conditionally in the way specified by the above iimit. Moreover. each integral aione is divergent, even conditionally. Had we considered them separately we would need to compensate that divergence around zero by subtracting some normalizing constants (for example, in the case $1<\alpha<2$, it would be just the expected value of the respective integral over $\left.(-\epsilon, \epsilon)^{c}\right)$. However, in our case there is no need for such a normalization since the two integrals $\int_{(-\epsilon, \epsilon)^{\mathrm{x}}} \int_{0}^{t} \int_{0}^{\infty}(\ldots)$ and $\int_{(-\epsilon, \epsilon)^{\mathrm{c}}} \int_{-x}^{0} \int_{0}^{\infty}(\ldots)$ have the same distribution and the normalization constants cancel.

In subsequent sections we show that the above process $\{X(t), t \geq 0\}$ is well defined, is $\alpha$-stable, has stationary increments and is self-affine with the exponent $H=(1-\theta) / \alpha$. We also analyse dependence structures and path properties, and consider more general puises.

\section{Existence}

Most of the properties of $\{X(t), t \geq 0\}$ as weil as its existence follow from the theory of stable integrals $\int_{E} f(x) M(\mathrm{~d} x)$, where $f$ is a non-random function and $M$ is an $\alpha$-stabie random measure. Let $(\Omega, \overline{\mathscr{F}}, P)$ be the underlying probability space and denote by $L^{0}(\Omega)$ the set of all real random

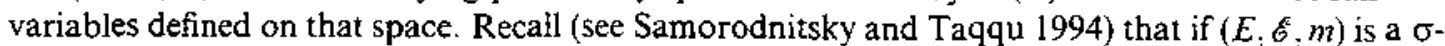
finite measure space, $\mathscr{E}_{0}=\{A \in \mathscr{E}: m(A)<\infty\}$, and $\beta: E \rightarrow[-1,1]$ is a measurable function, then an independently scattered $\sigma$-additive set function $M: \mathscr{E}_{0} \rightarrow L^{0}(\Omega)$ is called an $\alpha$-stable, $0<\alpha<2$. 
random measure on $(E, \mathscr{E})$ with control measure $m$ and skewness intensity $\beta$, if for each $A \in \mathscr{E}_{0}, M(A)$ is an $\alpha$-stable random variable with scale parameter $\{m(A)\}^{1 / \alpha}$, shift parameter 0 and skewness $\int_{A} \beta(x) m(\mathrm{~d} x) / m(A)$. Using standard procedures it is possible to construct stable stochastic integrals (obtained as limits in probability of integrals of simple functions) with respect to the measure $M$. A function $f$ is integrable if it satisfies $\int_{E}|f(x)|^{\alpha} m(\mathrm{~d} x)<\infty$ for $0<\alpha<2$, and additionally, $\int_{E}|f(x)(\ln |f(x)|) \beta(x)| m(\mathrm{~d} x)<\infty$ for $\alpha=1$. The resulting stable integral $\int_{E} f(x) M(\mathrm{~d} x)$ is a stable random variable with characteristic function

$$
\begin{aligned}
& \mathrm{E}\left[\exp \left\{\mathrm{i} t \int_{E} f(x) M(\mathrm{~d} x)\right\}\right]= \\
& \begin{cases}\exp \left\{-\int_{E}|t f(x)|^{\alpha}\left(1-\mathrm{i} \beta(x) \operatorname{sgn}(t f(x)) \tan \frac{\pi \alpha}{2}\right) m(\mathrm{~d} x)\right\}, & \text { if } \alpha \neq 1, \\
\exp \left\{-\int_{E}|t f(x)|\left(1+\mathrm{i} \frac{2}{\pi} \beta(x) \operatorname{sgn}(t f(x)) \ln |t f(x)|\right) m(\mathrm{~d} x)\right\}, & \text { if } \alpha=1 .\end{cases}
\end{aligned}
$$

The following lemma relates the integrais with respect to a Poisson random measure to those with respect to a stable measure. Its statement involves the constant

$$
C_{\alpha}= \begin{cases}\left(2 \alpha^{-1} \Gamma(1-\alpha) \cos (\pi \alpha / 2)\right)^{-1 / \alpha} & \text { if } 0<\alpha<1, \\ \left(-2 \alpha^{-1}(\alpha-1)^{-1} \Gamma(2-\alpha) \cos (\pi \alpha / 2)\right)^{-1 / \alpha} & \text { if } 1<\alpha<2, \\ 1 / \pi & \text { if } \alpha=1 .\end{cases}
$$

Lemma 2.1 (cf. Theorem 3.12.2 of Samorodnitsky and Taqqu 1994) Let $M$ be an $\alpha$-stable, $0<\alpha<2$, random measure on $(E, \mathscr{E})$ with control measure $m$ and skewness intensity $\beta$. Assume $m$ is $\sigma$-finite and $E=\cup_{i=1}^{\infty} E_{i}, E_{i} \in \mathscr{E}, m\left(E_{i}\right)<\infty$ and $E_{i} \cap E_{j}=\emptyset, i \neq j, i, j=1,2, \ldots$ Let $N$ be a Poisson random measure on $\left(\mathbb{R}_{0} \times E, \mathscr{B}\left(\mathbb{R}_{0}\right) \times \mathscr{E}\right)$ with intensity measure $n$ given by

$$
n(\mathrm{~d} \lambda, \mathrm{d} u)=\mathrm{E} N(\mathrm{~d} \lambda, \mathrm{d} u)= \begin{cases}\{1+\beta(u)\} \lambda^{-\alpha-1} \mathrm{~d} \lambda m(\mathrm{~d} u) & \text { if } \lambda>0, u \in E, \\ \{1-\beta(u)\}|\lambda|^{-\alpha-1} \mathrm{~d} \lambda m(\mathrm{~d} u) & \text { if } \lambda<0, u \in E .\end{cases}
$$

Finally, let $f$ be an integrable function. If $0<\alpha<1$, then

if $1<\alpha<2$, then

$$
\int_{E} f(u) M(\mathrm{~d} u) \stackrel{\mathrm{d}}{=} C_{\alpha} \int_{\mathbb{R}_{0}} \int_{E} \lambda f(u) N(\mathrm{~d} \lambda, \mathrm{d} u)
$$

$$
\int_{E} f(u) M(\mathrm{~d} u) \stackrel{\mathrm{d}}{=} C_{\alpha} \lim _{\epsilon \rightarrow 0} \sum_{i=1}^{\infty}\left(\int_{\{-\epsilon, \varepsilon)^{c}} \int_{E_{i}} \lambda f(u) N(\mathrm{~d} \lambda, \mathrm{d} u)-E \int_{\left(-\epsilon \in c^{c}\right.} \int_{E_{j}} \lambda f(u) N(\mathrm{~d} \lambda, \mathrm{d} u)\right) ;
$$

and if $\alpha=1$, then

$$
\begin{gathered}
\int_{E} f(u) M(\mathrm{~d} u) \\
\stackrel{\mathrm{d}}{=} C_{\alpha} \lim _{\epsilon \rightarrow 0} \sum_{i=1}^{\infty}\left(\int_{(-\epsilon, \epsilon)^{c}} \int_{E_{i}} \lambda f(u) N(\mathrm{~d} \lambda, \mathrm{d} u)-2 \ln \left\{\max \left(1, \epsilon^{-1}\right)\right\} \int_{E_{i}} f(u) \beta(u) m(\mathrm{~d} u)\right) \\
-2 b / \pi \int_{E} f(u) \beta(u) m(\mathrm{~d} u),
\end{gathered}
$$


where $b=\ln \pi+\int_{0}^{\infty}(\sin t-t I[t \leq 1]) t^{-2} \mathrm{~d} t$. The expressions in the right-hand sides of the above formulas converge a.s.

We are now able to infer the existence of the process.

Theorem 2.1. The process $\{X(t), t \geq 0\}$, given by (1.2), is well defined and its finite-dimensional distributions equal those of $\left\{C_{\alpha}^{-1} Y(t), t \geq 0\right\}$, where

$$
Y(t):=M\left(S_{0, t}^{+}\right)-M\left(S_{0, t}^{-}\right), \quad t \geq 0 .
$$

Here $M$ is an $\alpha$-stable random measure on $\left(\mathbb{R} \times \mathbb{R}_{+}, \mathscr{B}\left(\mathbb{R} \times \mathbb{R}_{+}\right)\right)$with constant skewness $\beta=\left(c^{\prime}-c^{\prime \prime}\right) /\left(c^{\prime}+c^{\prime \prime}\right)$ and control measure $m$ given by

$$
m(\mathrm{~d} \tau, \mathrm{d} w)=\frac{c^{\prime}+c^{\prime \prime}}{2} w^{-\theta-1} \mathrm{~d} \tau \mathrm{d} w
$$

for $\tau \in \mathbb{R}, w>0$, and

$$
\begin{aligned}
& S_{0, t}^{+}:=\{(\tau, w): 0<\tau<t, t-\tau<w\} \\
& S_{0, t}^{-}:=\{(\tau, w):-\infty<\tau<0,-\tau<w<t-\tau\} .
\end{aligned}
$$

Hence, the finite-dimensional distributions of $Y$ are $\alpha$-stable. The one-dimensional distributions are symmetric with scale parameter equal to $\left[\left(c^{\prime}+c^{\prime \prime}\right)\{\theta(1-\theta)\}^{-1} t^{1-\theta}\right]^{1 / \alpha}$. Moreover, the processes are self-affine with exponent $H=(1-\theta) / \alpha$ and have stationary increments, under the additional assumption $\beta=0$ for $\alpha=1$.

\section{Remark}

$S_{0, t}^{+}$involves the pulses whose time of birth is in $(0, t)$ and whose death occurs after $t$ and $S_{0, t}^{-}$involves the pulses which start before time 0 and die in $(0, t)$.

\section{Proof}

Consider first $Y(t)=M\left(S_{0, t}^{+}\right)-M\left(S_{0, r}^{-}\right)=\int_{\mathbb{R}^{\prime} \mathbb{R}_{-}}\left(f_{1}(\tau, w)-f_{2}(\tau, w)\right) M(\mathrm{~d} \tau, \mathrm{d} w)$, where $f_{1}$ and $f_{2}$ are the indicator functions of $S_{0, t}^{+}$and $S_{0, r}^{-}$, respectively. Since

$$
\begin{aligned}
\int_{S_{0, t}^{-}} m(\mathrm{~d} \tau, \mathrm{d} w) & =\int_{0}^{t} \int_{t-\tau}^{\infty}\left\{\left(c^{\prime}+c^{\prime \prime}\right) / 2\right\} w^{-\theta-1} \mathrm{~d} \tau \mathrm{d} w=\int_{-\infty}^{0} \int_{-\tau}^{t-\tau}\left(\left(c^{\prime}+c^{\prime \prime}\right) / 2\right) w^{-\theta-1} \mathrm{~d} \tau \mathrm{d} w \\
& =\int_{S_{0,}^{-}} m(\mathrm{~d} \tau, \mathrm{d} w)=\left(c^{\prime}+c^{\prime \prime}\right)\{2 \theta(1-\theta)\}^{-1} t^{1-\theta}<\infty,
\end{aligned}
$$

$\{Y(t), t \geq 0\}$ is a well-defined $\alpha$-stabie process, not necessarily symmetric. For fixed $t \geq 0$, however, $M\left(S_{0, t}^{+}\right)$and $M\left(S_{0, l}^{-}\right)$are independent and identically distributed random variables and therefore the one-dimensional distribution of $Y(t)$ is symmetric with scale parameter $\left(\left(c^{\prime}+c^{\prime \prime}\right)(\theta(1-\theta))^{-1} t^{1-\theta}\right)^{1 / \alpha}$. By Lemma 2.1 , the process $\{X(t), t \geq 0\}$ equals in distribution $\left\{C_{\alpha}^{-1} Y(t), t \geq 0\right\}$. Equality (2.5) explains why the compensating constants for $X(t), t \geq 0$, are zero for $\alpha \geq 1$. Moreover, although $m$ is only $\sigma$-finite (not finite) the fact that, for every $t$, both functions $f_{1}$ and $f_{2}$ are supported on $m$-finite sets (which again follows from the above formula) proves a.s. absolute convergence of each $\int_{(-\epsilon, \epsilon)^{c}} \int_{E} \lambda f_{i}(\tau, w) N(\mathrm{~d} \lambda, \mathrm{d} \tau, \mathrm{d} w), i=1,2$, separately, for $\alpha \geq 1, \epsilon>0$. 
Using (2.1), the joint characteristic function of $\left(Y_{t_{1}}, Y_{t_{2}}, \ldots, Y_{t_{n}}\right), t_{1}, t_{2}, \ldots, t_{n} \geq 0, n \geq 1$, for $\alpha \neq 1$, can be written as follows:

$$
\begin{gathered}
\mathrm{E}\left\{\exp \left(\mathrm{i} \sum_{j=1}^{n} \theta_{j} Y\left(t_{j}\right)\right)\right\}=\exp \left[-\int_{\mathbb{R} \times \mathbb{R}_{+}}\left|\sum_{j=1}^{n} \theta_{j}\left(I\left[S_{0, t_{j}}^{+}\right]-I\left[S_{0, t_{j}}^{-}\right]\right)\right|^{\alpha}\right. \\
\left.\times\left\{1-\mathrm{i} \beta \operatorname{sgn}\left(\sum_{j=1}^{n} \theta_{j}\left(I\left[S_{0, t_{j}}^{+}\right]-I\left[S_{0_{, j}}^{-}\right]\right)\right) \tan \frac{\pi \alpha}{2}\right\}\left(\left(c^{\prime}+c^{\prime \prime}\right) / 2\right) w^{-\theta-1} \mathbf{d} \tau \mathbf{d} w\right] .
\end{gathered}
$$

Note that, for $a, t>0$,

$$
\begin{aligned}
S_{0, a t}^{+} & =\{(\tau, w): 0<\tau<a t, a t-\tau<w\}=\{(\tau, w): 0<\tau / a<t, t-\tau / a<w / a\} \\
& =\left\{\left(a \tau^{\prime}, a w^{\prime}\right): 0<\tau^{\prime}<t, t-\tau^{\prime}<w^{\prime}\right\}=a S_{0, t}^{+}
\end{aligned}
$$

and, similarly, $S_{0, a t}^{-}=a S_{0, t}^{-}$. Hence, using the change of variables $\tau^{\prime}:=\tau / a, w^{\prime}:=w / a$, we get for $a, t_{1}, t_{2}, \ldots, t_{n} \geq 0, n \geq 1$,

$$
\mathrm{E}\left\{\exp \left(\mathrm{i} \sum_{j=1}^{n} \theta_{j} Y\left(a t_{j}\right)\right)\right\}=\mathrm{E}\left\{\exp \left(i \sum_{j=1}^{n} \theta_{j} a^{(1-\theta) / \alpha} Y\left(t_{j}\right)\right)\right\}
$$

that is,

$$
\{Y(a t), t \geq 0\} \stackrel{d}{=}\left\{a^{(1-\theta) / \alpha} Y(t), t \geq 0\right\},
$$

proving that $\{Y(t), t \geq 0\}$ is $(1-\theta) / \alpha$-self-affine. The proof for $\alpha=1$ is analogous.

The stationarity of increments can be proved in a similar fashion. Fix $b>0$ and $t_{1}, t_{2}, \ldots, t_{n} \geq 0$, $n \geq 1$. Again, consider only $\alpha \neq 1$. Then,

$$
\begin{gathered}
\mathrm{E}\left\{\exp \left(\mathrm{i} \sum_{j=1}^{n} \theta_{j}\left\{Y\left(t_{j}+b\right)-Y(b)\right\}\right)\right\}=\exp \left(-\int_{\mathbb{R} \times \mathbb{R}_{+}}\left|\sum_{j=1}^{n} \theta_{j}\left(I\left[S_{b, i_{j}+b}^{+}\right]-I\left[S_{b, t_{j}+b}^{-}\right]\right)\right|^{\alpha}\right) \\
\times\left\{1-\mathrm{i} \beta \operatorname{sgn}\left(\sum_{j=1}^{n} \theta_{j}\left(I\left[S_{b_{,} t_{j}+b}^{+}\right]-I\left[S_{b_{, j}+b}^{-}\right]\right)\right) \tan \frac{\pi \alpha}{2}\right\}\left\{\left(c^{\prime}+c^{n}\right) / 2\right\} w^{-\theta-1} \mathrm{~d} \tau \mathrm{d} w_{1}
\end{gathered}
$$

where

$$
\begin{aligned}
& S_{b, c}^{+}=\{(\tau, w): b<\tau<c, c-\tau<w\}, \\
& S_{b, c}^{-}=\{(\tau, w):-\infty<\tau<b, b-\tau<w<c-\tau\} .
\end{aligned}
$$

The simple translation, $\tau^{\prime}:=\tau-b$, shows that the above characteristic function equals that of $\left(Y\left(t_{1}\right), Y\left(t_{2}\right), \ldots, Y\left(t_{n}\right)\right)$, that is, that $\{Y(t), t \geq 0\}$ has stationary increments.

\section{Symmetric case and an extension to a self-affine random field with stationary increments}

When $\beta=0$, not only is $Y(t)$ symmetric, but all its finite-dimensional distributions are symmetric as 
well. In this case $\{Y(t), t \geq 0\}$ (and hence $\{X(t), t \geq 0\}$ ) is equivalent to a process introduced by Takenaka (1991). To see this, we need to make the following change of variables: $z:=\tau+w / 2$, $r:=w / 2$. Instead of defining a new $\alpha$-stable measure as the corresponding map of the measure $M$ in the definition of $\{Y(t), t \geq 0\}$, it is more useful for our purposes to use the following change-ofvariables lemma for Poisson random measures and obtain yet another version of the process $\{X(t), t \geq 0\}$.

Lemma 3.1 (Resnick 1987) Let $\phi: E \rightarrow E$ be a measurable mapping. If $N$ is a Poisson random measure on $(E, \mathscr{E})$ with intensity $n$, then $N^{\prime}$, defined by

$$
N^{\prime}(A):=N\left(\phi^{-1}(A)\right)
$$

for $A \in \mathscr{E}$ with $n\left(\phi^{-1}(A)\right)<\infty$, is a Poisson random measure on $(E, \mathscr{E})$ with intensity $n\left(\phi^{-1}(\cdot)\right)$. If we have a representation $N=\sum_{i} \delta_{X_{i}}$, then also $N^{t}=N \circ \phi^{-1}=\sum_{i} \delta_{\phi\left(X_{i}\right)}$. In particular,

$$
\int_{\phi^{-1}(A)} g(\phi(x)) N(\mathrm{~d} x)=\int_{A} g(y) N^{\prime}(\mathrm{d} y),
$$

whenever one of the sides is defined.

Let $\psi: \mathbb{R}_{0} \times \mathbb{R} \times \mathbb{R}_{+} \rightarrow \mathbb{R}_{0} \times \mathbb{R} \times \mathbb{R}_{+}$be defined as

$$
\psi(\lambda, \tau ; w)=(\lambda, \tau+w / 2, w / 2)
$$

If $N$ is a Poisson random measure with intensity given by (1.1), then $N^{\prime}:=N \circ \psi^{-1}$ is a Poisson measure with intensity

$$
n^{\prime}(\mathrm{d} \lambda, \mathrm{d} z, \mathrm{~d} r)= \begin{cases}2^{-\theta} c^{\prime} \lambda^{-\alpha-1} r^{-\theta-1} \mathrm{~d} \lambda \mathrm{d} z \mathrm{~d} r & \text { if } \lambda>0, \\ 2^{-\theta} c^{\prime \prime}|\lambda|^{-\alpha-1} r^{-\theta-1} \mathrm{~d} \lambda \mathrm{d} z \mathrm{~d} r & \text { if } \lambda<0\end{cases}
$$

for $z \in \mathbb{R}$ and $r>0$. The process $\left\{X^{t}(t), t \geq 0\right\}$, defined as the difference of integrals with respect to $N^{\prime}$ in the same way as $\{X(t), t \geq 0\}$ in (1.2), but with $w$ and $\tau$ replaced by $2 r$ and $z-r$ (cf. also (3.5) below), is of course equivalent to $\{Y(t), t \geq 0\}$. On the other hand, by Lemma 2.1,

$$
\left\{X^{\prime}(t), t \geq 0\right\} \stackrel{d}{=} \text { const. }\left\{M^{\prime}\left(S_{0 . t}^{+}\right)-M^{\prime}\left(S_{0 . t}^{\prime *}\right), t \geq 0\right\}
$$

where $M^{\prime}$ is an $\alpha$-stable measure on $\left(\mathbb{R} \times \mathbb{R}_{+}, \mathscr{B}\left(\mathbb{R} \times \mathbb{R}_{+}\right)\right)$with constant skewness $\beta=\left(c^{\prime}-c^{\prime \prime}\right) /\left(c^{\prime}+c^{\prime \prime}\right)$ and control measure $m^{\prime}, m^{\prime}(\mathrm{d} z, \mathrm{~d} r)=2^{-\theta-1}\left(c^{\prime}+c^{\prime \prime}\right) r^{-\theta-1} \mathrm{~d} z \mathrm{~d} r$ for $z \in \mathbb{R}$. $r>0$, and

$$
\begin{aligned}
& S_{0, t}^{\prime+}=\{(z, r): r>t-z, 0<z-r<t\}=\{(z, r):|z-t|<r,|z|>r\}, \\
& S_{0, r}^{\prime-}=\{(z, r):-z<r<t-z, z-r<0\}=\{(z, r):|z-t|<r,|z|>r\} .
\end{aligned}
$$

When $\beta=0$, that is $c^{\prime}=c^{\prime \prime}, M^{\prime}$ is symmetric and hence $M^{\prime}(\cdot) \stackrel{\text { d }}{=}-M^{\prime}(\cdot)$. Moreover, $M^{\prime}\left(S_{0, t_{j}}^{+}\right)$. $j=1,2, \ldots, n, n \geq 1$, are jointiy independent from $M^{\prime}\left(S_{0, t_{f}}^{-}\right), j=1,2, \ldots, n, n \geq 1$, since 
$S_{0, t_{j}}^{+} \cap S_{0, t_{k}}^{-}=\emptyset$ for all choices of $t_{1}, \ldots, t_{n}$. Hence,

$$
\begin{aligned}
& \left(M^{\prime}\left(S_{0, t_{1}}^{\prime+}\right)-M^{\prime}\left(S_{0, f_{1}}^{\prime-}\right), M^{\prime}\left(S_{0, t_{2}}^{+}\right)-M^{\prime}\left(S_{0, t_{2}}^{\prime-}\right), \ldots, M^{\prime}\left(S_{0, t_{n}}^{\prime+}\right)-M^{\prime}\left(S_{0, t_{n}}^{\prime-}\right)\right) \\
& =\left(M^{\prime}\left(S_{0, t_{1}}^{+}\right), M^{\prime}\left(S_{0, t_{2}}^{+}\right), \ldots, M^{\prime}\left(S_{0, t_{n}}^{+}\right)\right)-\left(M^{\prime}\left(S_{0, t_{1}}^{-}\right), M^{\prime}\left(S_{0, t_{2}}^{-}\right), \ldots, M^{\prime}\left(S_{0, t_{n}}^{-}\right)\right) \\
& \stackrel{\mathrm{d}}{=}\left(M^{\prime}\left(S_{0, t_{1}}^{+}\right), M^{\prime}\left(S_{0, t_{2}}^{++}\right), \ldots, M^{\prime}\left(S_{0, t_{n}}^{+}\right)\right)+\left(M^{\prime}\left(S_{0, t_{1}}^{\prime-}\right), M^{\prime}\left(S_{0, t_{2}}^{\prime-}\right), \ldots, M^{\prime}\left(S_{0, t_{n}}^{-}\right)\right) \\
& =\left(M^{\prime}\left(S_{0, l_{1}}^{\prime+}\right)+M^{\prime}\left(S_{0, l_{1}}^{\prime-}\right), \ldots, M^{\prime}\left(S_{0, l_{n}}^{\prime+}\right)+M\left(S_{0, l_{n}}^{\prime-}\right)\right) \\
& =\left(M^{\prime}\left(S_{0, t_{1}}^{\prime+} \cup S_{0, t_{1}}^{-}\right), \ldots, M^{\prime}\left(S_{0, t_{n}}^{\prime+} \cup S_{0, t_{n}}^{-}\right)\right) \text {. }
\end{aligned}
$$

Since, for $t \geq 0$,

$$
S_{0, t}^{+} \cup S_{0, t}^{-}=\{(z, r):|z-t|<r\} \Delta\{(z, r):|z|<r\}
$$

where $\Delta$ denotes symmetric difference, we see, that in the symmetric case $\beta=0$, the process $\{X(t), t \geq 0\}$ is a version of the process

$$
\left\{M^{\prime}\left(S_{0, t}^{\prime+} \cup S_{0, t}^{\prime-}\right), t \geq 0\right\}=\left\{M^{\prime}\left(S_{0, t}^{\prime+}\right)+M^{\prime}\left(S_{0, i}^{\prime-}\right), t \geq 0\right\}
$$

introduced by Takenaka. Relations (3.2) and (3.3) differ by the sign in their right-hand sides. The choice of sign plays no role in the symmetric case. In the non-symmetric case, however, it is essential that the sign be minus (as in (3.2)), because otherwise the process would not have stationary increments. Thus the pulses not only provide the physical construction of the process but also indicate what the correct sign ought to be.

There is a generalization of the above process to the situation where the time parameter is multidimensional. That is, we will construct a self-affine random field with stationary increments. In the symmetric case such a random field was constructed by Takenaka. Our alternative construction, besides being applicable to a non-symmetric case as well, allows us to view the resulting random fields as superposition of multidimensional puises.

Let $M^{\prime}$ denote an $\alpha$-stable measure on $\left(\mathbb{R}^{d} \times \mathbb{R}_{+}, \mathscr{B}\left(\mathbb{R}^{d} \times \mathbb{R}_{+}\right)\right)$with constant skewness $\beta$ and control measure $m^{\prime}, m^{\prime}(\mathrm{dz}, \mathrm{d} r)=r^{-\theta-d} \mathrm{dz} \mathrm{d} r$ for $\mathrm{z} \in \mathbb{R}^{d}, r>0$. Then

$$
\left\{M^{\prime}\left(S_{0, \mathrm{t}}^{+}\right)-M^{\prime}\left(S_{0, \mathrm{t}}^{-}\right), \mathrm{t} \in \mathbb{R}^{d}\right\}
$$

with

$$
\begin{aligned}
& S_{0, \mathrm{t}}^{++}=\left\{(\mathbf{z}, r):\|\mathbf{z}-\mathbf{t}\|<r_{:}\|\mathbf{z}\|>r\right\}, \\
& S_{0, \mathrm{t}}^{--}=\{(\mathbf{z}, r):\|\mathbf{z}-\mathbf{t}\|>r,\|\mathbf{z}\|<r\},
\end{aligned}
$$

is an $\alpha$-stable $(1-\theta) / \alpha$-self-affine random field with stationary increments.

In the analogous definition of Takenaka there is, of course, a plus sign in (3.4) instead of a minus sign. He interprets $S_{0, \mathrm{t}}^{\prime+} \cup S_{0, \mathrm{t}}^{\prime-}$ as the set of all $(d-1)$-dimensional spheres separating points $\mathbf{0}$ and $\mathbf{t}$. Variables $\mathrm{z}$ and $r$ denote the centre and the radius of a sphere, respectively. It seems that in order to obtain a 'proper' measure of the separating spheres (that is, one which also works in non-symmetric cases) one should subtract (not add) the measure of spheres containing zero from the measure of spheres containing point $\mathbf{t}$.

Now let us return to our original construction. The change of variables $\psi$ can be interpreted in the following way. If $(\lambda, \tau, w)$ is a pulse starting at time $\tau$, with height $\lambda$ and duration $w$, then $z=\tau+w / 2$ is the centre of the pulse and $r=w / 2$ its radius. These variables are particularly useful in the $\mathbb{R}^{d}$ generalization. Note that (3.4) is equivalent in distribution (up to a multiplicative 
constant) to $\left\{X^{\prime}(\mathbf{t}), \mathbf{t} \in \mathbb{R}^{d}\right\}$ defined by

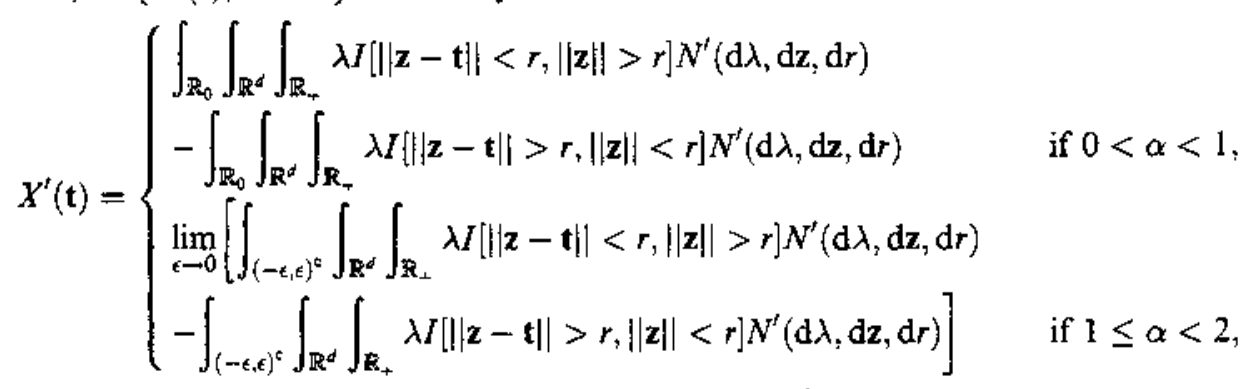

where $N^{\prime}$ is a Poisson random measure on $\mathbb{R}_{0} \times \mathbb{R}^{d} \times \mathbb{R}_{+}$with intensity $n^{\prime}(\mathrm{d} \lambda, \mathrm{d} \boldsymbol{z}, \mathrm{d} \mathbf{r})=$ $(1 \pm \beta)|\lambda|^{-\alpha-1} \mathrm{~d} \lambda m^{\prime}(\mathrm{d} \mathbf{z}, \mathrm{d} r)$ if $\pm \lambda>0$. This interpretation of $X^{\prime}(t), t \geq 0$, as a sum of pulses still holds. Now a pulse is a cylinder in $\mathbb{R}^{\alpha+1}$ space. It is described by its height $(\lambda)$, radius $(r)$ and the centre of its circular base (z). But the only pulses which really count are those whose bases contain either point 0 or point $t$ (but not both). To get $X^{\prime}(t)$ one adds the pulses containing $t$ in their base and subtracts those that contain 0 in their base.

\section{Dependence structure}

It follows from the definition (2.3) (or (3.2)) of the process $\{Y(t), t \geq 0\}$ that its two-dimensional distributions determine its multidimensional structure. More precisely, given the definition of the process as a difference of measures (or integrals) of the sets $S_{0, t}^{-}$and $S_{0, t}^{-}$, knowledge of all twodimensional distributions suffices to specify any multidimensional distribution; this fact does not depend on the particular choice of $m$. To verify it, first notice that it is enough to know multidimensional distributions of non-overlapping increments. On the other hand, these can be described using intersections of at most two sets of the type $S_{b, c}^{+}, S_{b, c}^{-}$(cf. (2.6)) or their complements, and they, in turn, are specified by two-dimensional characteristic functions. For the proof in the $d$ dimensional symmetric case look at Sato (1991), where it is shown that the finite-dimensional distributions of (3.4) are determined by $(d+1)$-dimensional marginals.

We may conclude that the dependence structure of the (one-dimensional) process $\{Y(t), t \geq 0\}$ resembies that of Gaussian processes. To develop this point, we need to examine the characteristic function of two non-overlapping increments and compare their interrelation to the covariance in the Gaussian case.

We will need the following general form of the characteristic function of an $\alpha$-stable vector $(X, Y)$ :

$$
\left\{\begin{array}{cc}
\operatorname{E}\left[\exp \left\{\mathrm{i}\left(\xi_{1} X+\xi_{2} Y\right)\right\}\right]= \\
\exp \left\{-\int_{S_{2}}\left|\xi_{1} s_{1}+\xi_{2} s_{2}\right|^{\alpha}\left(1-\mathrm{i} \operatorname{sgn}\left(\xi_{1} s_{1}+\xi_{2} s_{2}\right) \tan \frac{\pi \alpha}{2}\right) \Gamma(\mathrm{ds})\right. & \text { if } \alpha \neq 1 \\
\left.+\mathrm{i}\left(\xi_{1} \mu_{1}+\xi_{2} \mu_{2}\right)\right\} & \\
\exp \left\{-\int_{S_{2}}\left|\xi_{1} s_{1}+\xi_{2} s_{2}\right|\left(1+\mathrm{i} \frac{2}{\pi} \operatorname{sgn}\left(\xi_{1} s_{1}+\xi_{2} s_{2}\right) \ln \left|\xi_{1} s_{1}+\xi_{2} s_{2}\right|\right) \Gamma(\mathrm{ds})\right. & \\
\left.+\mathrm{i}\left(\xi_{1} \mu_{1}+\xi_{2} \mu_{2}\right)\right\} & \text { if } \alpha=1
\end{array}\right.
$$


where the unique finite measure $\Gamma$ (called the spectral measure) is defined on Borel sets of the unit circle $S_{2}$ and $\mu_{1}, \mu_{2} \in \mathbb{R}$ are the location parameters.

Below we calculate the characteristic function of the vector $(Y(s), Y(u)-Y(t)), 0<s<t<u$, which is obviously equal to that of $(Y(s+h)-Y(h), Y(u+h)-Y(t+h))$ for any $h>0$, because $\{Y(t), t \geq 0\}$ has stationary increments. (We assume $\beta=0$ when $\alpha=1$, in order to ensure stationarity of the increments in this case as well.) In order to obtain the characteristic function we partition the sets $S_{0, s}^{+}, S_{0, s}^{-}, S_{t, u}^{+}, S_{t, u}^{-}$and consider independent variables

$$
M\left(S_{0, s}^{-}\right), M\left(S_{t, u}^{+}\right), M\left(S_{0, s}^{+} \cap S_{t, u}^{-}\right), M\left(S_{0, s}^{+} \backslash S_{t, u}^{-}\right), M\left(S_{t, u}^{-} \backslash S_{0, s}^{+}\right),
$$

whose respective scale parameters satisfy (see (2.5)):

$$
\begin{aligned}
\sigma^{\alpha}\left(M\left(S_{0, s}^{-}\right)\right) & =\left(c^{\prime}+c^{\prime \prime}\right)\{2 \theta(1-\theta)\}^{-1} s^{1-\theta} ; \\
\sigma^{\alpha}\left(M\left(S_{t, u}^{+}\right)\right) & =\left(c^{\prime}+c^{\prime \prime}\right)\{2 \theta(1-\theta)\}^{-1}(u-t)^{1-\theta} \\
\sigma^{\alpha}\left(M\left(S_{0, s}^{+} \cap S_{t, u}^{-}\right)\right) & =\left(c^{\prime}+c^{\prime \prime}\right)\{2 \theta(1-\theta)\}^{-1}\left\{t^{1-\theta}-u^{1-\theta}-(t-s)^{1-\theta}+(u-s)^{1-\theta}\right\} \\
\sigma^{\alpha}\left(M\left(S_{0, s}^{+} \backslash S_{t, u}^{-}\right)\right) & =\left(c^{\prime}+c^{\prime \prime}\right)\{2 \theta(1-\theta)\}^{-1}\left\{s^{1-\theta}-t^{1-\theta}+u^{1-\theta}+(t-s)^{1-\theta}-(u-s)^{1-\theta}\right\} \\
\sigma^{\alpha}\left(M\left(S_{t, u}^{-} \backslash S_{0, s}^{+}\right)\right) & =\left(c^{\prime}+c^{\prime \prime}\right)\{2 \theta(1-\theta)\}^{-1}\left\{(u-t)^{1-\theta}-t^{1-\theta}+u^{1-\theta}+(t-s)^{1-\theta}-(u-s)^{1-\theta}\right\} .
\end{aligned}
$$

Hence,

$$
\begin{aligned}
& \mathrm{E}\left\{\exp \left(\mathrm{i}\left[\xi_{1} Y(s)+\xi_{2}\{Y(u)-Y(t)\}\right]\right)\right\} \\
& =\mathrm{E}\left(\operatorname { e x p } \left[\mathrm { i } \left\{\xi_{1} M\left(S_{0, s}^{+} \backslash S_{i, u}^{-}\right)-\xi_{1} M\left(S_{0, s}^{-}\right)+\xi_{2} M\left(S_{t, u}^{+}\right)-\xi_{2} M\left(S_{i, u}^{-} \backslash S_{0, s}^{+}\right)\right.\right.\right. \\
& \left.\left.\left.+\left(\xi_{1}-\xi_{2}\right) M\left(S_{0, s}^{+} \cap S_{i, u}^{-}\right)\right\}\right]\right) \\
& =\exp \left\{-\left(c^{\prime}+c^{\prime \prime}\right)(2 \theta(1-\theta))^{-1}\left(\left|\xi_{1}\right|^{\alpha}\left[2 s^{1-\theta}-t^{1-\theta}+u^{1-\theta}+(t-s)^{1-\theta}-(u-s)^{1-\theta}\right]\right.\right. \\
& \left(1-\mathbf{i} \beta\left[2 s^{1-\theta}-t^{1-\theta}+u^{i-\theta}+(t-s)^{1-\theta}-(u-s)^{1-\theta}\right]^{-1}\right. \text {. } \\
& \left.\left[-t^{1-\theta}+u^{1-\theta}+(t-s)^{1-\theta}-(u-s)^{1-\theta}\right] \operatorname{sgn} \xi_{1} \tan \frac{\pi \alpha}{2}\right) \\
& +\left|\xi_{2}\right|^{\alpha}\left[2(u-t)^{1-\theta}-t^{\mathrm{I}-\theta}+u^{1-\theta}+(t-s)^{1-\theta}-(u-s)^{1-\theta}\right. \\
& \left(1-\mathrm{i} \beta\left[2(u-t)^{1-\theta}-t^{1-\theta}+u^{1-\theta}+(t-s)^{1-\theta}-(u-s)^{1-\theta}\right]^{-1}\right. \\
& {\left[t^{1-\theta}-u^{1-\theta}-(t-s)^{1-\theta}+(u-s)^{1-\theta} \mid \operatorname{sgn} \xi_{2} \tan \frac{\pi \alpha}{2}\right)} \\
& \left.\left.+\left|\xi_{1}-\xi_{2}\right|^{\alpha}\left[t^{1-\theta}-u^{1-\theta}-(t-s)^{1-\theta}+(u-s)^{1-\theta}\right]\left(1-\mathrm{i} \beta \operatorname{sgn}\left(\xi_{1}-\xi_{2}\right) \tan \frac{\pi \alpha}{2}\right)\right)\right\} \\
& = \begin{cases}\exp \left\{-\int_{S_{2}}\left|\xi_{1} s_{1}+\xi_{2} s_{2}\right|^{\alpha}\left(1-\mathrm{i} \operatorname{sgn}\left(\xi_{1} s_{1}+\xi_{2} s_{2}\right) \tan \left(\frac{\pi \alpha}{2}\right) \Gamma(\mathbf{d s})\right)\right\} & \text { if } \alpha \neq 1, \\
\exp \left\{-\int_{S_{2}}\left|\xi_{1} s_{1}+\xi_{2} S_{2}\right| \Gamma(\mathrm{ds})\right\} & \text { if } \alpha=1 .\end{cases}
\end{aligned}
$$


where

$$
\begin{aligned}
\Gamma= & \left(c^{\prime}+c^{\prime \prime}\right)\{2 \theta(1-\theta)\}^{-1}\left\{\left(s^{1-\theta}-\frac{1+\beta}{2}\left(t^{1-\theta}-u^{1-\theta}-(t-s)^{1-\theta}+(u-s)^{1-\theta}\right)\right) \delta_{1,0}\right. \\
& +\left(s^{1-\theta}-\frac{1-\beta}{2}\left(t^{1-\theta}-u^{1-\theta}-(t-s)^{1-\theta}+(u-s)^{1-\theta}\right)\right) \delta_{(-1,0)} \\
& +\left((u-t)^{1-\theta}-\frac{1-\beta}{2}\left(t^{1-\theta}-u^{1-\theta}-(t-s)^{1-\theta}+(u-s)\right)^{1-\theta}\right) \delta_{(0,1)} \\
& +\left((u-t)^{1-\theta}-\frac{1+\beta}{2}\left(t^{1-\theta}-u^{1-\theta}-(t-s)^{1-\theta}+(u-s)\right)^{1-\theta}\right) \delta_{(0,-1)} \\
& \left.+\left(t^{1-\theta}-u^{1-\theta}-(t-s)^{1-\theta}+(u-s)^{1-\theta}\right) 2^{\alpha / 2}\left(\frac{1+\beta}{2} \delta_{(\sqrt{2} / 2,-\sqrt{2} / 2)}+\frac{1-\beta}{2} \delta_{(-\sqrt{2} / 2, \sqrt{2} / 2)}\right)\right\}
\end{aligned}
$$

Now, one would like to describe the dependence structure between two increments using some analogue of the autocorrelation function. Unfortunately, in the stable non-Gaussian case, there is no single function which could play the role of the covariance. Indeed, it is the whole spectral measure $\Gamma$ which gives the information about the interrelation between two variables. Nevertheless, there are known measures of dependence such as the covariation and the codifference which partially replace the covariance when $0<\alpha<2$.

Given two jointly $\alpha$-stable variables $X$ and $Y$ with spectral measure $\Gamma$, the covariation of $X$ and $Y$ equals

$$
[X, Y]:=\int_{S_{2}} s_{1} s_{2}^{\langle\alpha-1\rangle} \Gamma(\mathrm{ds})
$$

where $a^{\langle\beta\rangle}:=|a|^{\beta} \operatorname{sgn} a$ for $a, \beta \in \mathbb{R}$. One of the obvious flaws of the covariation is that it is not symmetric in its arguments. Another is that it is generally not defined for $\alpha \leq 1$.

Recently Kokoszka and Taqqu (1994) introduced the notion of codifference for jointly symmetric $\alpha$-stable variables $X$ and $Y$. We extend their definition to the non-symmetric case by considering a symmetrization of the vector $(X, Y)$, that is, the codifference of $X$ and $Y$ equals

$$
\begin{aligned}
\tau(X, Y): & =\operatorname{Re} \ln \mathrm{E}[\exp \{\mathrm{i}(X-Y)\}]-\operatorname{Re} \ln \mathrm{E}\{\exp (\mathrm{i} X)\}-\operatorname{Re} \ln \mathrm{E}\{\exp (\mathrm{i} Y)\} \\
& =-\int_{S_{2}}\left|s_{1}-s_{2}\right|^{\alpha} \Gamma(\mathrm{ds})+\int_{S_{2}}\left|s_{1}\right|^{\alpha} \Gamma(\mathrm{ds})+\int_{S_{2}}\left|s_{2}\right|^{\alpha} \Gamma(\mathrm{d} s) .
\end{aligned}
$$

Note that in the Gaussian case $(\alpha=2)$, we have

$$
\tau(X, Y)=2[X, Y]=2[Y, X]=\operatorname{cov}(X, Y) .
$$

For the increments of process $\{Y(t), t \geq 0\}$ we get

$$
\begin{aligned}
\tau(Y(s), Y(u)-Y(t)) & =\left(2^{\alpha}-2\right)[Y(s), Y(u)-Y(t)]=\left(2^{\alpha}-2\right)[Y(u)-Y(t), Y(s)] \\
& =-\left(2^{\alpha}-2\right) 2^{-\alpha / 2}\{\Gamma(\sqrt{2} / 2,-\sqrt{2} / 2)+\Gamma(-\sqrt{2} / 2, \sqrt{2} / 2)\} \\
& =-\left(2^{\alpha}-2\right)\left(c^{\prime}+c^{\prime \prime}\right)\{2 \theta(1-\theta)\}^{-1}\left\{t^{1-\theta}-u^{1-\theta}-(t-s)^{1-\theta}+(u-s)^{1-\theta}\right\} .
\end{aligned}
$$


Before interpreting this result, note that in our case the spectral measure $\Gamma$ has exceptionally simple form. It is clear that the dependence between $Y(s)$ and $Y(u)-Y(t)$ is due to the mass $\Gamma$ gives to the points $\pm(\sqrt{2} / 2,-\sqrt{2} / 2)$. Hence, any function pretending to extend the covariance must make use of mass of $\Gamma$ concentrated at these points. Equivalently, one may want to keep in mind the following unique (up to the equality in distribution) representation of the increments (cf. (4.1)):

$$
(Y(s), Y(u)-Y(t)) \stackrel{d}{=}\left(U_{1}+V, U_{2}-V\right)
$$

where $U_{1}, U_{2}$ and $V$ are independent $\alpha$-stable variables with $V$, the random variable which affects the dependence, having skewness $\beta$ and the $\alpha$ th power of its scale parameter equal to

$$
\left(c^{\prime}+c^{\prime \prime}\right)\{2 \theta(1-\theta)\}^{-1}\left\{t^{1-\theta}-u^{1-\theta}-(t-s)^{1-\theta}+(u-s)^{1-\theta}\right\} .
$$

Then we are able to define a measure of dependence between $Y(s)$ and $Y(u)-Y(t)$ as the $\alpha$ th power of the scale parameter of the 'common' variable $V$. To get an analogue of the correlation, let us normalize it by the product of the scale parameters of the increments raised to the power $\alpha / 2$; that is, let us consider the function

$$
r(s, t, u):=-\frac{t^{1-\theta}-u^{t-\theta}-(t-s)^{1-\theta}+(u-s)^{1-\theta}}{2 \sqrt{s^{1-\theta}(u-t)^{1-\theta}}} .
$$

We put the minus sign to underline the negative dependence of the two increments. Hence, for $1<\alpha<2$, the normalized covariation (that is, divided by $([Y(s), Y(s)][Y(u)-$ $Y(t), Y(u)-Y(t)]^{1 / 2}=\left(\sigma^{\alpha}(Y(s)) \sigma^{\alpha}(Y(u)-Y(t))\right)^{1 / 2}$ ) equals exactly $r(s, t, u)$. (One could also consider $\alpha=1$ with $a^{(0)}=\operatorname{sgn} a$. The covariation is not defined here for $a<1$.) Also the codifference $\tau(Y(s), Y(u)-Y(t))$, for $\alpha \neq 1$, properly normalized, equals $r(s, t, u)$. For $0<\alpha \leq 1$, on the other hand, it seems here that $r(s, t, u)$ measures dependence in a more proper way than the codifference. For $\alpha=1$, the codifference becomes zero, although the increments are far from being independent, and for $0<\alpha<1$ the codifference is positive $\left(2^{\alpha}-2<0\right)$.

We should remark that all preceding normalized measures of dependence equal the correlation of the respective increments of the fractional Brownian motion with the self-affine exponent $H^{\prime}=H \alpha / 2=(1-\theta) / 2$ (cf. Mandelbrot and Van Ness 1968). For example, the measure of dependence $r$ for one-step increments, $k$ steps apart, takes a very well-known form:

$$
r(1, k, k+1)=\left\{(k+1)^{1-\theta}+(k-1)^{1-\theta}-2 k^{1-\theta}\right\} / 2 \text {. }
$$

In the case $H^{t}=(1-\theta) / 2<\frac{1}{2}$, that is, $H=(1-\theta) / \alpha<1 / \alpha$, the dependence of the increments of fractional Brownian motion is antipersistent and we extend this terminology to the dependence structure of process $\{Y(t), t \geq 0\}$. Note that our construction does not allow for $H^{\prime}>\frac{1}{2}(H>1 / \alpha)$ which corresponds to persistent (or positive) long-run dependence.

Clearly, it is the special nature of the process $\{Y(t), t \geq 0\}$ which allows the above measures of dependence to coincide. For most processes $r(s, t, u)$ cannot be even defined. However, whenever the representation $X(t)=\int_{A_{t}} M(\mathrm{~d} u)=M\left(A_{t}\right), t \in T$, holds for some $M$, then a 'good' measure of dependence between $X\left(t_{1}\right)$ and $X\left(t_{2}\right)$ (or even $\left.X\left(t_{1}\right), X\left(t_{2}\right), \ldots, X\left(t_{n}\right)\right)$ should be

$$
\int_{A_{t_{1}} \cap A_{t_{2}}} m(\mathrm{~d} t)=m\left(A_{t_{1}} \cap A_{t_{2}}\right)
$$

(or $m\left(\cap_{i=1}^{n} A_{t_{t}}\right)$ ) properly normalized. 


\section{More general processes}

Let $0<\alpha<2$, and let $F: \mathbb{R}^{k} \rightarrow \mathbb{R}_{+}$be a function satisfying the two following relations:

$$
F\left(c x_{1}, c x_{2}, \ldots, c x_{k}\right)=\mathcal{c}^{-(1+8)} F\left(x_{1}, x_{2}, \ldots, x_{k}\right)
$$

for $c>0, x_{1}, x_{2}, \ldots, x_{k} \in \mathbb{R}$, and fixed $\theta \in \mathbb{R}$; and

$$
F\left(x_{1}+t, x_{2}+t, \ldots, x_{k}+t\right)=F\left(x_{1}, x_{2}, \ldots, x_{k}\right)
$$

for $t, x_{1}, x_{2}, \ldots, x_{k} \in \mathbb{R}$.

Let $N$ be a Poisson random measure on $\left(\mathbb{R}_{0} \times \mathbb{R}^{k}, \mathscr{B}\left(\mathbb{R}_{0} \times \mathbb{R}^{k}\right)\right)$ with intensity

$$
n\left(\mathrm{~d} \lambda, \mathrm{d} x_{1}, \ldots, \mathrm{d} x_{n}\right)=|\lambda|^{-\alpha-1} F\left(x_{1}, \ldots, x_{k}\right) \mathrm{d} \lambda \mathrm{d} x_{1} \ldots, \mathrm{d} x_{k}
$$

for $\lambda \neq 0, x_{1}, \ldots, x_{k} \in \mathbb{R}$. Put $Z(0) \equiv 0$ and

$$
Z(t):=\int_{\mathbb{R}_{0}} \int_{\mathbb{R}^{k}} \lambda \sum_{i=1}^{k} a_{i} I\left[0<x_{i} \leq t\right] N\left(\mathrm{~d} \lambda, \mathrm{d} x_{1}, \ldots, \mathrm{d} x_{k}\right)
$$

for $t>0$, where $a_{i} \in \mathbb{R}, i=1,2, \ldots, k$.

The integral in (5.3) is well defined for every $t>0$ (possibly only in the sense of conditional convergence if $\alpha \geq 1$ ) if

$$
\int_{\mathbb{R}^{k}}\left|\sum_{i=1}^{k} a_{i} I\left[0<x_{i} \leq t\right]\right|^{\alpha} F\left(x_{1}, \ldots, x_{n}\right) \mathrm{d} x_{1} \ldots \mathrm{d} x_{n}<\infty
$$

for every $t>0$. In this case $\{Z(t), t \geq 0\}$ is a symmetric $\alpha$-stable process, which, up to a multiplicative constant, has the same finite-dimensional distributions as

$$
Z^{\prime}(t)=\int_{\mathbb{R}^{k}} \sum_{i=1}^{k} a_{i} I\left[0<x_{i} \leq t\right] M\left(\mathrm{~d} x_{1}, \ldots, \mathrm{d} x_{k}\right),
$$

where $M$ is a $\mathrm{S} \alpha \mathrm{S}$ random measure on $\left(\mathbb{R}^{k}, \mathscr{B}\left(\mathbb{R}^{k}\right)\right)$ with control measure $m\left(\mathrm{~d} x_{1}, \ldots, \mathrm{d} x_{n}\right)=$ $F\left(x_{1}, \ldots, x_{n}\right) \mathrm{d} x_{1} \ldots \mathrm{d} x_{n}$. (We could consider Poisson measure $N$ with non-symmetric intensity $n$. Then (5.3) may require compensating for $Z(t)$ in order to converge. The measure $M$ and process $\{Z(t), t \geq 0\}$ would be, in general, non-symmetric.)

It is easy to check that, under conditions (5.1), (5.2) and (5.4), $\left\{Z^{\prime}(t), t \geq 0\right\}$ (hence also $\{Z(t), t \geq 0\})$ is $(k-1-\theta) / \alpha$-self-affine and has stationary increments.

When $k=1$, any function $F$ satisfying (5.1) and (5.2) must be constant and the process $\{Z(t), t \geq 0\}$ is, in fact, $\alpha$-stable Lévy motion (with independent increments).

When $k=2, F\left(x_{1}, x_{2}\right)=\left|x_{2}-x_{1}\right|^{-1-\theta} F\left(0, \operatorname{sgn}\left(x_{2}-x_{1}\right)\right)$ and if at least one of $F(0,1), F(0,-1)$ is positive, then (5.4) implies

$$
\begin{aligned}
\infty & >\int_{\mathbb{R}} \int_{\mathbb{R}}\left|a_{1} I\left[0<x_{1} \leq t\right\}+a_{2} I\left[0<x_{2} \leq t\right]\right|^{\alpha}\left(x_{2}-x_{1}\right)^{-1-\theta} I\left[x_{2}>x_{1}\right] \mathrm{d} x_{1} \mathrm{~d} x_{2} \\
& =\int_{\mathbb{R}} \int_{\mathbb{R}}\left|a_{1} I[0<\tau \leq t]+a_{2} I[-\tau<w \leq t-\tau]\right|^{\alpha} n^{,-1-\theta} I[w>0] \mathrm{d} \tau \mathrm{d} w .
\end{aligned}
$$

The above inequality holds only when $a_{1}+a_{2}=0$ and $0<\theta<1$. We recognize that in the latter 
case the process $\{Z(t), t \geq 0\}$ is equivalent (up to a multiplicative constant) to processes obtained in the previous sections, in particular to $\{X(t), t \geq 0\}$ given by (1.2). The variables $x_{1}$ and $x_{2}$ have simple interpretation as the moment of pulse birth (that is, $\tau$ ) and the moment of puise death $(\tau+w)$, respectively, if $x_{2}>x_{1}$, and vice versa if $x_{1}>x_{2}$.

The following example shows that there is an $F$ with properties (5.1) and (5.2) in the case $k=3$. It can be easily extended to $k>3$. Let

$$
F\left(x_{1}, x_{2}, x_{3}\right)=\left(x_{2}-x_{1}\right)^{-\theta_{1}-1}\left(x_{3}-x_{2}\right)^{-\theta_{2}-1} I\left[\epsilon_{1}<\frac{x_{3}-x_{2}}{x_{2}-x_{1}}<\frac{1}{\epsilon_{2}}\right] I\left[x_{1}<x_{2}<x_{3}\right],
$$

where $1 / \epsilon_{2}>\epsilon_{1}>0$. Then, if $a_{i} \neq 0, i=1,2,3$, it is necessary (and sufficient, too) that $0<\theta_{1}+\theta_{2}<1$ and $a_{1}+a_{2}+a_{3}=0$ for (5.4) to hold. We may still interpret $Z(t), t \geq 0$, as the difference in the total magnitude of pulses existing at time $t$ and at time 0 , but now the shape of a pulse is different. It consists of three, or in general $k$, jumps with the last one equal to the negative of the sum of all previous jumps $\left(a_{k}=-\left(a_{1}+\cdots+a_{k-1}\right)\right)$. The ith jump occurs at time $x_{i}$ and at this time the height of the pulse changes by $\lambda a_{i}$. The resulting height (i.e. $\lambda \sum_{j=1}^{i} a_{j}$ ) is maintained for a period of time $w_{i}=x_{i+1}-x_{i}>0$. Note that we cannot take the variables $w_{i}$ independent and distributed as $w_{i}^{-\theta_{i}-1} \mathrm{~d} w_{i}$ with $\theta_{i}>0$, since the convolution of every two such measures would be infinite and so would be the width of a puise. (This explains the presence of the indicator $I\left[\epsilon_{1}<\left(x_{3}-x_{2}\right) /\left(x_{2}-x_{1}\right)<1 / \epsilon_{2}\right]$ in the above definition of $F$.) On the other hand, keeping the $x_{i}$ in increasing order is just a useful simplification. It should be clear from (5.3), or from (5.5), that integrals over regions with different orderings of $x_{i}$ give independent contributions to $Z(t)$. In the geometric in terpretation, these integrals correspond to adding pulses of various shapes with $k$ jumps whose size is proportional to the same $k$ numbers $a_{i}$, but the jumps occur in different order. Because such sums of pulses are independent and have a similar structure, we shall focus on one particular order of $a_{i}$ or $x_{i}$

Let us also notice that the $a_{i}$ (or rather their ratios) determine the dependence structure of multidimensional distributions of $\{Z(t), t \geq 0\}$, that is, they determine points on the muitidimensional sphere where the spectral measure lives. Both $F$ and $a_{i}$ determine the mass at these points. Since $k$ jumps may affect at most $k$ different disjoint increments of $Z(t)$ and the $a_{i}$ are fixed, knowledge of all $k$-dimensional distributions of $\{Z(t), t \geq 0\}$ suffices to describe this process (refer to the discussion at the beginning of Section 4 for $k=2$ ). Moreover, in the case $k \geq 3$, when $\sum_{i=1}^{k} a_{i}=0$, two kinds of dependence between two non-overlapping increments appear - positive, corresponding to pulses either increasing or decreasing in both time intervals, and negative when the opposite is true. For $k=2\left(a_{1}=-a_{2}\right)$ we have only negative dependence (see Section 4).

No matter how big $k$ is, however, the dependence structure of multidimensional distributions of $\{Z(t), t \geq 0\}$ is simple in the sense that the corresponding spectral measures are discrete. If we randomize the $a_{i}$ we may obtain non-discrete spectral measures. Then the integral (5.5) will no longer be a linear combination of random measure of some sets. However, the interpretation of $Z(t)$ as a sum of pulses becomes less clear since the shape of the pulses is not fixed, but is a function itself. As an exampie, for $k=3$, consider

$$
\begin{aligned}
Z(t)= & \int_{\mathbb{R}_{0}} \int_{\mathbb{R}^{3}} \int_{0}^{2 \pi} \lambda\left\{\sin \phi\left(I\left[0<x_{1} \leq t\right]-I\left[0<x_{3} \leq t\right]\right)\right. \\
& \left.+\cos \phi\left(I\left[0<x_{2} \leq t\right]-I\left[0<x_{3} \leq t\right]\right)\right\} N\left(\mathrm{~d} \lambda, \mathrm{d} x_{1}, \mathrm{~d} x_{2}, \mathrm{~d} x_{3}, \mathrm{~d} \phi\right)
\end{aligned}
$$


where the Poisson measure $N$ on $\left(\mathbb{R}_{0} \times \mathbb{R}^{3} \times[0,2 \pi), \mathscr{B}\left(\mathbb{R}_{0} \times \mathbb{R}^{3} \times[0,2 \pi)\right)\right.$ has intensity

$$
n\left(\mathrm{~d} \lambda, \mathrm{d} x_{1}, \mathrm{~d} x_{2}, \mathrm{~d} x_{3}, \mathrm{~d} \phi\right)=|\lambda|^{-\alpha-1} F\left(x_{1}, x_{2}, x_{3}\right) \mathrm{d} \lambda \mathrm{d} x_{1} \mathrm{~d} x_{2} \mathrm{~d} x_{3} \mathrm{~d} \phi
$$

with $F$ as in (5.6). Each pulse consists of jumps of height $\lambda \sin \phi, \lambda \cos \phi$ and $-\lambda(\sin \phi+\cos \phi)$. Summing pulses, also over $\phi$, indeed, gives a continuous component of spectral measures for twodimensional distributions.

\section{Remark on sample path behaviour}

The sample path behaviour of the original process (1.2), or equivalently its version (2.3), is very irregular. We will show that sampie paths are nowhere bounded, which contradicts the statement made by Kôno and Maejima (1991) about the Takenaka process.

We will use the following facts (see Samorodnitsky and Taqqu 1994, Corollary 9.5.5 and Theorem 10.2.3). A stable process is sample bounded with positive probability if and only if it is sample bounded with probability 1 . Moreover, if an $\alpha$-stable process with an integral representation $\left\{\int_{E} f(t, u) M(\mathrm{~d} u), t \in T\right\}$ is sample bounded then, necessarily,

$$
\sup _{T \subset T} \int_{E} \sup _{t \in T}|f(t, u)|^{\alpha} m(\mathrm{~d} u)<\infty
$$

where $T^{*}$ is any countable subset of $T$.

Consider process $\{Y(t), t \geq 0\}$ given by (2.3). We will prove that

$$
\left.\int_{\mathbb{R}} \int_{\mathbb{R}_{+}+\in T^{*}} \sup _{0} I S_{0, t}^{+}\right] w^{-\theta-1} \mathrm{~d} \tau \mathrm{d} w=\infty
$$

for $T^{*}=\mathbb{Q} \cap I$, where $\mathbb{Q}$ is the set of rational numbers and $I$ is a finite interval in $(0, \infty)$. Hence. $\{Y(t), t \geq 0\}$ is unbounded on every finite interval, that is, nowhere bounded, with probability 1 .

To establish (6.1) consider the indicator $I\left[S_{0, t}^{+}\right]=I[0<\tau<t, t-\tau<w]$ as a function of $t$ with fixed $\tau$ and $w$. Then $I\left[S_{0, t}^{+}\right]=1$ if and only if $\tau<t<w+\tau$. Fix an interval $l$ and note that for any $(\tau, w), \tau \in I$ and $w>0$, there exists $t_{0} \in T^{*}=\mathbb{Q} \cap I$ such that $\tau<t_{0}<\tau+w$. Thus,

$$
\begin{aligned}
\int_{\mathbb{R}} \int_{\mathbf{R}_{+}} \sup _{t \in T^{*}} I\left[S_{0, t}^{+}\right] w^{-\theta-1} \mathrm{~d} \tau \mathrm{d} w & \geq \int_{I \times \mathbb{R}_{-} t \in T^{*}} \sup I\left[S_{0, t}^{+}\right] w^{-\theta-1} \mathrm{~d} \tau \mathrm{d} w \\
& =\int_{I \times \mathbb{R}_{+}} w^{-\theta-1} \mathrm{~d} r \mathrm{~d} w=\infty .
\end{aligned}
$$

Similar reasoning proves that also the process $\left\{Z^{\prime}(t), t \geq 0\right\}$ defined by (5.5) with $k=3$ and $F$ given by (5.6) is nowhere bounded with probability 1 . In this case the statement follows, for example, from the fact that

$$
\begin{gathered}
\int_{\mathbb{R}^{3}} \sup _{i \in T^{+}}\left|a_{1} I\left[0<x_{1}<t<x_{2}<x_{3}\right]\right|^{\alpha} F\left(x_{1}, x_{2}, x_{3}\right) \mathrm{d} x_{1} \mathrm{~d} x_{2} \mathrm{~d} x_{3} \\
=\left|a_{1}\right|^{\alpha} \int_{\mathbb{R}} \int_{\mathbb{R}_{+}} \int_{\mathbb{R}_{+}} \sup _{t \in T^{+}} I\left[0<\tau<t<\tau+w_{1}<\tau+w_{2}\right] w_{1}^{-\theta_{1}-1} w_{2}^{-\theta_{2}-1} I\left[\epsilon_{1}<\frac{w_{2}}{w_{1}}<\frac{1}{\epsilon_{2}}\right] \mathrm{d} \tau \mathrm{d} w_{1} \mathrm{~d} w_{2} \\
\geq\left|a_{1}\right|^{\alpha} \int_{I} \int_{\mathbb{R}_{+}} \int_{\mathbb{R}_{+}} w_{1}^{-\theta_{1}-1} w_{2}^{-\theta_{2}-1} I\left[\epsilon_{1}<\frac{w_{2}}{w_{1}}<\frac{1}{\epsilon_{2}}\right] \mathrm{d} \tau \mathrm{d} w_{1} \mathrm{~d} w_{2}=\infty .
\end{gathered}
$$




\section{Acknowledgement}

The third and fourth authors were partially supported by Office of Naval Research Grant N0001490-J-1287 at Boston University.

\section{References}

Cioczek-Georges, R. and Mandelbrot, B.B. (1994a) A class of micropulses and antipersistent fractional Brownian motion. Stoch. Process. Appl. To appear.

Cioczek-Georges, R. and Mandelbrot, B.B. (1994b) Alternative micropulses and FBM. Preprint.

Cioczek-Georges, R. and Mandelbrot, B.B. (1995) Stable fractal sums of pulses: the general case. Preprint.

Itô, K. (1969) Stochastic Processes, Vol. 16, Lecture Notes Series, Mathematics Institute, Aarhus University. Kokoszka, P.S. and Taqqu, M.S. (1994) Infinite variance stable ARMA processes. J. Time Ser. Anal., 15, 203220.

Kôno, N. and Maejima, M. (1991) Setf-similar stable processes with stationary increments. In S. Cambants, G. Samorodnitsky and M.S. Taqqu (eds). Stable Processes and Related Topics, Vol. 25 of Progress in Probability, pp. 275-295. Boston: Birkhäuser.

Lovejoy. S. and Mandelbrot, B.B. (1985) Fractal properties of rain, and a fractal model. Tellus, 37(A), 209232.

Mandelbrot, B.B. (1984) Fractal sums of pulses, and new random variabies and functions. Unpublished.

Mandelbrot, B.B. (1995a) Introduction to fractal sums of pulses. In G. Zaslawsky. M.F. Schlesinger and U. Frisch (eds), Lévy Flights and Related Phenomena (Nice, 1994), Lecture Notes in Physics. New York: Springer-Verlag.

Mandelbrot, B.B. (1995b) Some fractai sums of pulses: affine convolutions, giobal dependence, and a versatile family of self-affine random functions. Preprint.

Mandetbrot, B.B. and Van Ness, J.W. (1968) Fractiona! Brownian motions, fractional noises and applications. SIAM Rev., 10, 422-437.

Mori, T. and Sato, Y. (1994) Construction and asymptotic behavior of a class of self-similar stable processes. Preprint.

Resnick, S.I. (1987) Extreme Values, Regular Variation and Point Processes. New York: Springer-Verlag.

Samorodnitsky, G and Taqqu, M.S. (1994) Stable Non-Gaussian Processes: Stochastic Models with Infinite Variance. London: Chapman \& Hall.

Sato, Y. (1992) Structure of bivariate distributions of linear functional stable and Chentsov type stable processes. In A.N. Shiryaev, V.S. Krolyuk, S. Watanabe and M. Fukushima (eds), Probability Theory and Mathematical Statistics, Proceedings of the Sixth USSR-Japan Symposium, pp. 306-316. Singapore: World Scientific.

Sato, Y. (1991) Distributions of stable random fields of Chentsov type. Nagoya Math. J., 123, 119-139.

Takenaka, S. (1991) Integral-geometric construction of self-similar stable processes. Nagoya Math. J., 123, $1-12$.

Received May 1994 and revised October 1994 